\title{
From "tough on crime" to "smart on crime"
}

$\mathrm{T}$ This fall, members of Canada's Parliament will consider Bill C-15, which would impose mandatory minimum prison terms for certain drug offences involving any quantity of controlled substances, such as heroin, cocaine and amphetamines. Bill C-15 sounds the bell for yet another round of "get tough on crime" rhetoric. Interestingly, after 30 years our neighbour to the south has finally begun detoxifying from this policy and is beginning to adopt the "get smart on crime" mantra. Canadians can get smart on crime now and avoid an unimaginable public health disaster.

As Bill C-15 is currently drafted, the most marginalized people living with addictions and/or living in poverty, who are engaged in small-scale trafficking (often related to their drug dependence), will be ensnared by the mandatory incarceration provisions. Incarcerating untold numbers of people for minor drug offenses is counterproductive to protecting the public's health and violates human rights.

Evidence indicates that imprisoning people who inject drugs fans the flames of Canada's HIV epidemic. The HIV prevalence rate in Canadian prisons is at least 10 times that found in the population as a whole. ${ }^{1}$ Furthermore, incarceration has been shown to lead to injection drug use among some incarcerated people who did not previously use drugs or use by injection. Correctional Services of Canada acknowledges that drugs enter prisons despite its efforts and that an estimated $80 \%$ of people in federal penitentiaries have substance-use problems. ${ }^{2}$ However, those who need sterile injection equipment do not yet have it increasing the risk of HIV transmission. And the vast majority of incarcerated m people eventually return to their communities, taking with them any infections they acquired while imprisoned.

Some policymakers are starting to respond to the public demand that drug addiction be addressed more as a health concern than a strictly criminal matter.

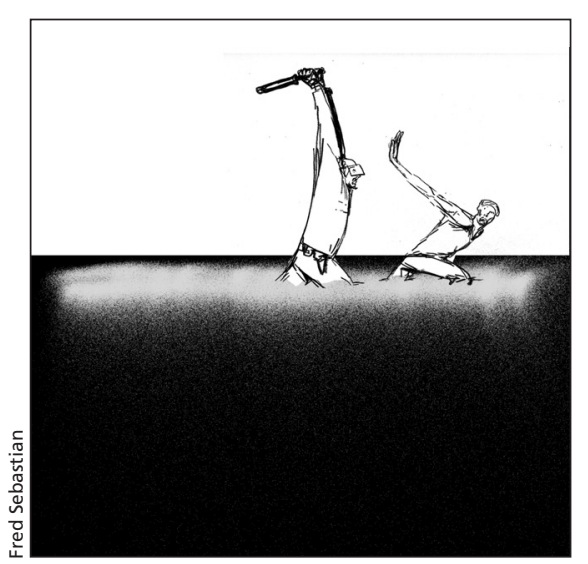

In 1998, Canada established its first drug treatment court - an effort toward diverting people from prison. Since then, the federal government has fervently poured resources into expanding these courts.

Not surprisingly, Bill C-15 calls for the use of these courts as a way to avoid incarcerating those who desperately need help with their addiction. While diverting people struggling with addiction away from prison is certainly a sensible public policy approach, the Canadian drug court initiative is actually a quick-fix that has been inadequately evaluated; its over emphasis on punishment hinders the ability to treat drug addiction as a health problem. This is because these courts merely suspend the criminal axe, which will come down with a vengeance, forcing an individual back to criminal count and, ultimately to prison should they fail to conform to the abstinence model: the treatment protocol currently afforded through these courts.

Three critical goals drive the impetus for the expansion of drug treatment courts: reduction of recidivism and drug use, cost-effectiveness and decreased pressure on correctional services. Thus far, the 2 evaluations conducted on Canadian treatment courts show that they fail to meet these critical goals. One vital shortcoming is the failure of these court programs to adequately assess people's drug dependence, pre- and post-drug treatment program. ${ }^{3}$ The current abstinence-based model also appears to either exclude or expel individuals dealing with more virulent drug dependence. For instance, the evaluation of the Toronto drug treatment court revealed that of the total participants (365) only $15.6 \%$ graduated from the drug treatment program, the rest were either expelled or withdrew. ${ }^{4}$ The coercion (a choice between treatment or jail) and abstinence model may be the root cause of the high failure rate experienced in the Toronto and Vancouver drug courts. ${ }^{3}$

Research-based evidence shows that prisons are breeding grounds for the transmission of HIV and hepatitis C; diverting people from prison to treatment is likely to improve public health - provided treatment programs effectively address people's diverse health needs. As Canadians are swept into another round to tackle addiction, they are well positioned to thoughtfully develop an approach more centred on health.

\section{Patricia Allard LLB}

Deputy Director

Canadian HIV/AIDS Legal Network

Toronto, Ont.

\section{REFERENCES}

1. Lines R, Jürgens R, Betterridge G, et al. Prison needle exchange: lessons from a comprehensive review of international evidence and experience. 2nd ed. Toronto (ON): Canadian HIV/AIDS Legal Network; 2006.

2. Corrections fast facts - drugs in prisons. Ottawa $(\mathrm{ON})$ : Public Safety and Emergency Preparedness Canada; 2007.

3. Werb D, Elliott R, Fischer B, et al. Drug treatment courts in Canada: an evidence-based review. HIV/AIDS Policy \& Law Review 2007;12(2/3):1217.

4. Public Safety Canada. Toronto drug treatment court evaluation project final report. Toronto (ON): National Crime Prevention Centre; 2007.

The Canadian HIV/AIDS Legal Network works on legal and human rights issues related HIV/AIDS.

Have you got an opinion about this article? Post your views at www.cmaj.ca.

All editorial matter in CMAJ represents the opinions of the authors and not necessarily those of the Canadian Medical Association. 